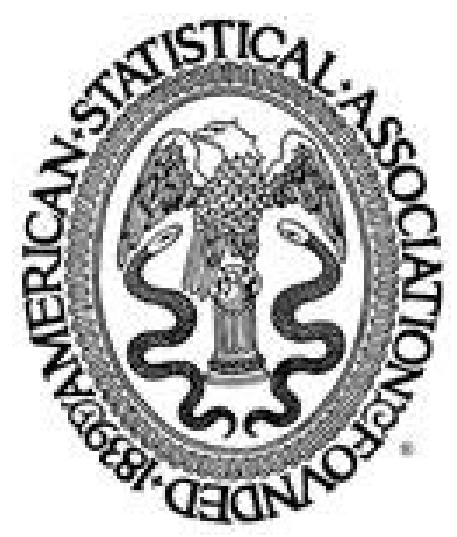

American Educational Research Association

Estimation of Unattenuated Factor Loadings

Author(s): Todd S. Woodward and Michael A. Hunter

Source: Journal of Educational and Behavioral Statistics, Vol. 24, No. 4 (Winter, 1999), pp. 384 $-397$

Published by: American Educational Research Association and American Statistical Association

Stable URL: http://www.jstor.org/stable/1165369

Accessed: $13 / 01 / 2009$ 15:49

Your use of the JSTOR archive indicates your acceptance of JSTOR's Terms and Conditions of Use, available at http://www.jstor.org/page/info/about/policies/terms.jsp. JSTOR's Terms and Conditions of Use provides, in part, that unless you have obtained prior permission, you may not download an entire issue of a journal or multiple copies of articles, and you may use content in the JSTOR archive only for your personal, non-commercial use.

Please contact the publisher regarding any further use of this work. Publisher contact information may be obtained at http://www.jstor.org/action/showPublisher?publisherCode=aera.

Each copy of any part of a JSTOR transmission must contain the same copyright notice that appears on the screen or printed page of such transmission.

JSTOR is a not-for-profit organization founded in 1995 to build trusted digital archives for scholarship. We work with the scholarly community to preserve their work and the materials they rely upon, and to build a common research platform that promotes the discovery and use of these resources. For more information about JSTOR, please contact support@jstor.org.

American Educational Research Association and American Statistical Association are collaborating with JSTOR to digitize, preserve and extend access to Journal of Educational and Behavioral Statistics. 


\title{
Estimation of Unattenuated Factor Loadings
}

\author{
Todd S. Woodward \\ Michael A. Hunter \\ University of Victoria
}

Keywords: exploratory factor analysis, unattenuated factor loadings, common factor analysis, principal component analysis

It is demonstrated that traditional exploratory factor analytic methods, when applied to correlation matrices, cannot be used to estimate unattenuated factor loadings. However, these values can be accurately estimated when the disattenuated correlation matrix, or the covariance matrix, is used as input. A mathematical basis for the advantage of this application of factor analysis is presented in this paper, as is an explanation of how these equations apply differentially to common factor analysis (CFA) and principal component analysis (PCA). Graphic displays which describe the comparative performance of CFA and PCA when extracting factors from the correlation matrix, the covariance matrix, and the disattenuated correlation matrix are provided. It is concluded that although the most accurate estimates of the unattenuated factor loadings can be achieved when CFA is used to decompose the covariance matrix or the disattenuated correlation matrix as the percentage of measurement error decreases, and the number of indicators per factor increases, the impact of methodology choice diminishes.

The 90-year history of exploratory factor analysis has focused primarily on methods designed to estimate correlations between observed scores and latent factors. We would like to raise an issue that we believe has previously been neglected; namely, the utility of exploratory factor analytic methods for estimating correlations between true scores and latent factors. Although the disattenuation of correlations between observed scores and factors is occasionally put into practice (e.g., Ree \& Earles, 1992), a detailed discussion promoting informed decision making, based on data characteristics, has been unavailable. The purpose of the present paper is to provide a framework for understanding the interaction between attenuation due to measurement error and choice of factor analytic methodology.

Expansion of a set of equations published by Widaman $(1990,1993)$ can provide a basis for such a framework. Widaman derived expressions providing a mathematical basis for the difference in population factor loadings defined by common factor analysis (CFA) and principal component analysis (PCA) for certain special cases. With these results, he showed that the differences in dimension reduction techniques pivot on their accuracy in estimating the communalities (a variable's communality is defined as the part of its variance that 
can be explained by the latent factors). By generalizing these expressions to incorporate attenuation attributable to measurement error, the three-way interaction between factor analytic technique (CFA vs. PCA), input matrix, and data set characteristics (viz., unattenuated factor loading size, percentage of measurement error, and number of indicators per factor) can be demonstrated, and the cause for these methodology differences specified.

\section{Attenuated Common Variance}

Traditionally, exploratory CFA has been conceptualized as a decomposition of the correlation matrix (e.g., Kaiser, 1970). The common factor model can be specified as follows (based on Widaman, 1993, p. 267):

$$
{ }_{p} R_{p}-{ }_{p} U_{p}^{2} \cong{ }_{p} A_{r} \Phi_{r} A_{p}^{\prime}={ }_{p} R_{p}{ }^{*},
$$

where $R$ represents the matrix of correlations among the observed-score variables, $U^{2}$ the diagonal matrix of unique variance, $A$ the rotated factor pattern matrix, $\Phi$ the matrix of factor intercorrelations, and $R^{*}$ the matrix of reproduced correlations among the observed variables ( $p$ is the number of variables, and $r$ the number of factors). Unique variance can be divided into specific and error variance (e.g., Gorsuch, 1983, p. 10; Nunnally \& Bernstein, 1994, p. 466). Specific variance is true-score variance that is not related to the latent factors, and error variance is measurement error, or error-score variance. In contrast to CFA, PCA does not distinguish between common and unique variance, but apportions all variance in the correlation matrix to the factor pattern matrix. That is to say, PCA represents a special case of the common factor model where zeros lie on the diagonal of the $U^{2}$ matrix of Equation (1).

We now introduce terminology that reflects the distinction between the values that are estimates of the communalities, and the unknown (except in simulation studies) values that represent the communalities being estimated (i.e., the variance of a variable that is attributable to the latent factors). These will be termed the estimated communalities and the attenuated communalities, respectively. The sum of the estimated communalities will be termed the estimated common variance, and the sum of the attenuated communalities will be termed the attenuated common variance.

We use the adjective attenuated to emphasize the fact that the size of the attenuated communalities and the attenuated common variance depend, in a compensatory fashion, on the true proportion of measurement error in the data set. That is to say, the influence of the latent factors remains constant over measurement conditions, but the proportion of variance in a variable that is sensitive to that influence varies with measurement quality. Thus, if a variable's variance is composed almost entirely of measurement error, the proportion of a variable's variance attributable to the latent factors (i.e., the attenuated communality) will be greatly reduced. 


\section{Unattenuated Common Variance}

A true-score based statistic that is often of interest to psychologists is the true-score correlation between two tests. To estimate this value from observedscore information, one can apply Spearman's (1904) correction for attenuation:

$$
\rho_{T_{X} T_{Y}}=\frac{\rho_{X Y}}{\sqrt{\rho_{X X^{\prime}} \rho_{Y Y^{\prime}}}},
$$

where $\rho_{T_{X} T_{Y}}$ represents the correlation between the true score for test $X$ and the true score for test $Y, \rho_{X Y}$ the correlation between observed scores $X$ and $Y$, and $\rho_{X X^{\prime}}$, and $\rho_{Y Y^{\prime}}$ reliabilities of observed scores $X$ and $Y$. Note that Equation (2) could be used either to disattenuate observed correlations between variables and factors, or to disattenuate observed correlations that are to be used as input for dimension reduction. In other words, dividing the factor loadings by the square root of the corresponding variable's reliability would produce identical results to disattenuating the input correlation matrix. However, focus on this post hoc application of Spearman's correction for attenuation formula does not allow an understanding of the interaction between factor analytic technique, data characteristics, and input matrix, as does a focus on differences in communality estimation. Thus, for pedagogical purposes, the present analysis focuses on the effect of observed correlation disattenuation, and the subsequent variance and covariance partitions, as opposed to post-hoc loading disattenuation.

When the correlation matrix is accurately disattenuated, the influence of measurement error is removed. Under these conditions, the $U^{2}$ term in the common factor model (Equation [1]) would be composed of only specific variance. The proportion of a variable's variance attributable to the latent factors when the influence of measurement error is not present will be referred to as the unattenuated communality. If they were discoverable, the unattenuated communalities could be summed over the variables-this sum will be termed the unattenuated common variance.

Unlike correlations, the covariances of obtained scores are equal to the covariances of true scores (Nunnally \& Bernstein, 1994, p. 240). Therefore, basing the common factor model on a covariance matrix is similar to basing it on a disattenuated correlation matrix, in that the matrix resulting from $R-U^{2}$ (see Equation [1]) would be composed only of unattenuated common variance. In summary, when a covariance matrix or a disattenuated correlation matrix are used as input for CFA, the rotated pattern matrix $A$ would provide accurate estimates of the unattenuated correlations or covariances between the observed variables and the factors (in the case of orthogonal factors), as opposed to attenuated correlations. Although the accuracy of CFA under these conditions is straightforward, the performance of PCA, and that of CFA when the data is contaminated by measurement error is not. The consequence of input matrix/factor analytic methodology choice can be specified by expansion of Widaman's $(1990,1993)$ equations. 


\section{Common Factor Analysis Versus Principal Component Analysis}

Debate over the comparative virtues of CFA and PCA for estimating the factor loading matrix has followed much of the course of the technique's history (e.g., Thurstone, 1935, p. 130, criticized the principal components method of Hotelling, 1933). Interestingly, in this decade there has been a resurgence of this debate (e.g., Bentler \& Kano, 1990; Bookstein, 1990; Fava \& Velicer, 1992; Gorsuch, 1990; Mulaik, 1990; Rozeboom, 1990; Velicer \& Jackson, 1990a, 1990b; Widaman, 1990, 1993). This historical exchange has been effectively quieted by Widaman (1993), who concluded that "the prudent researcher should rarely, if ever, opt for a component analysis of empirical data if his/her goal were to interpret the patterns of observed covariation among variables as arising from latent variables or factors" (p. 308).

Widaman (1993) showed mathematically that the differences between these dimension reduction techniques pivot on their ability to estimate the variable communalities. He writes that the

inaccuracy in representing parameters of a common factor solution should always be rather less than would occur in a component solution, because commonly used communality estimates are virtually always more accurate estimates of communality than is unity, the value used in component analysis (p. 271).

Communalities are not explicitly represented in the common factor model, but would lie on the diagonal of the matrix resulting from $R-U^{2}$ (see Equation [1]). As Widaman did, we will ignore the effect of sampling error in our discussions, and assume that the entire population has been measured.

\section{Equation Development}

Equations based on those presented by Widaman (1993), which elucidate the implications of using alternative matrices for input to dimension reduction procedures, will now be introduced. In order to directly expand upon Widaman's equations, the following restrictions must carry over to our analysis: the loading sizes were uniform, the number of indictors per factor were uniform, a variable had a non-zero correlation with one factor only, and factors were orthogonal (i.e., the $\Phi$ matrix of Equation [1] was the identity matrix) and rotated to simple structure (i.e., the $A$ matrix of Equation [1] was a rotated factor pattern matrix). Although Widaman's equations, and our extensions of them, are based on factor loadings resulting from a rotated solution, identical conclusions would be reached when observing other analysis stages sensitive to differences in common variance estimation (e.g., comparison of unrotated solutions, or direct comparison of eigenvalues).

We assume that, as is consistently demonstrated in simulation studies, CFA accurately estimates the attenuated communalities (e.g., Borgatta, Kercher, \& Stull, 1986; McArdle, 1990; Snook \& Gorsuch, 1989; Velicer \& Fava, 1987; Velicer, Peacock, \& Jackson, 1982; and Widaman, 1990, 1993), even under 
conditions of few indicators per factor (Borgatta et al., 1986; McArdle, 1990; Snook \& Gorsuch, 1989; Widaman, 1990, 1993). Finally, because various CFA methods produce equally accurate communality estimates (e.g., Browne, 1968; McArdle, 1990), we use CFA as an umbrella term encompassing the various CFA methods.

Under these conditions, CFA loadings are determined if the estimated communalities are known. All CFA loadings will equal the square root of the communality, because factors explain variance for one variable only. However, to facilitate comparison to PCA, we present a more detailed method for calculating CFA loadings. The first step is to calculate the CFA estimated common variance, which is equal to the sum of the estimated communalities:

$$
\hat{\boldsymbol{\sigma}}_{\text {CFAcom }}^{2}=\sum_{j=1}^{p} \hat{h}_{j}^{2}
$$

Where $\hat{h}_{j}^{2}$ is the estimated communality for variable $j$.

The total estimated common variance attributable to one factor is $\hat{\sigma}_{C F A c o m}^{2} / r$, where $r$ is the number of factors. When this variance is split over the indicators, we arrive at the CFA loadings squared. Therefore, the estimated squared CFA loadings may be calculated as:

$$
\hat{\lambda}_{\text {CFA }}^{2}=\left(\hat{\sigma}_{\text {CFAcom }}^{2} / r\right) / m,
$$

where $m$ is the number of indicators per factor.

PCA loadings can also be determined mathematically by using a variant of Equation (4); however, the $\hat{\sigma}_{C F A c o m}^{2}$ term cannot be used. This is because each of the $p$ initially extracted components explains a fraction $(1 / p)$ of the estimated unique variance (i.e., the sum of the diagonal of the estimated $U^{2}$ matrix). The unique variance interpreted as common by each PCA component is determined by:

$$
\hat{\sigma}_{u c o m}^{2}=\frac{1}{p}\left(\sigma_{\text {total }}^{2}-\hat{\sigma}_{\text {CFAcom }}^{2}\right),
$$

where $\sigma_{\text {total }}^{2}$ is the total (summed) variance of the variables. The estimated common variance that will be redistributed over the final PCA loadings by can be calculated as:

$$
\hat{\sigma}_{\text {PCAcom }}^{2}=\hat{\sigma}_{\text {CFAcom }}^{2}+r \hat{\sigma}_{\text {ucom }}^{2} .
$$

When the value of $\hat{\sigma}_{P C A c o m}^{2}$ is then entered into Equation (4) in place of $\hat{\sigma}_{C F A c o m}^{2}$ (note that $\hat{\sigma}_{P C A c o m}^{2}$ will be greater than $\hat{\sigma}_{C F A c o m}^{2}$ whenever measurement error or specific variance is present), the squared estimated PCA loadings can be calculated as:

$$
\hat{\lambda}_{P F A}^{2}=\left(\hat{\sigma}_{P C A c o m}^{2} / r\right) / m \text {. }
$$

Thus, PCA loadings are determined if the CFA estimated communalities, the number of factors, and the number of indicators per factor are known. 


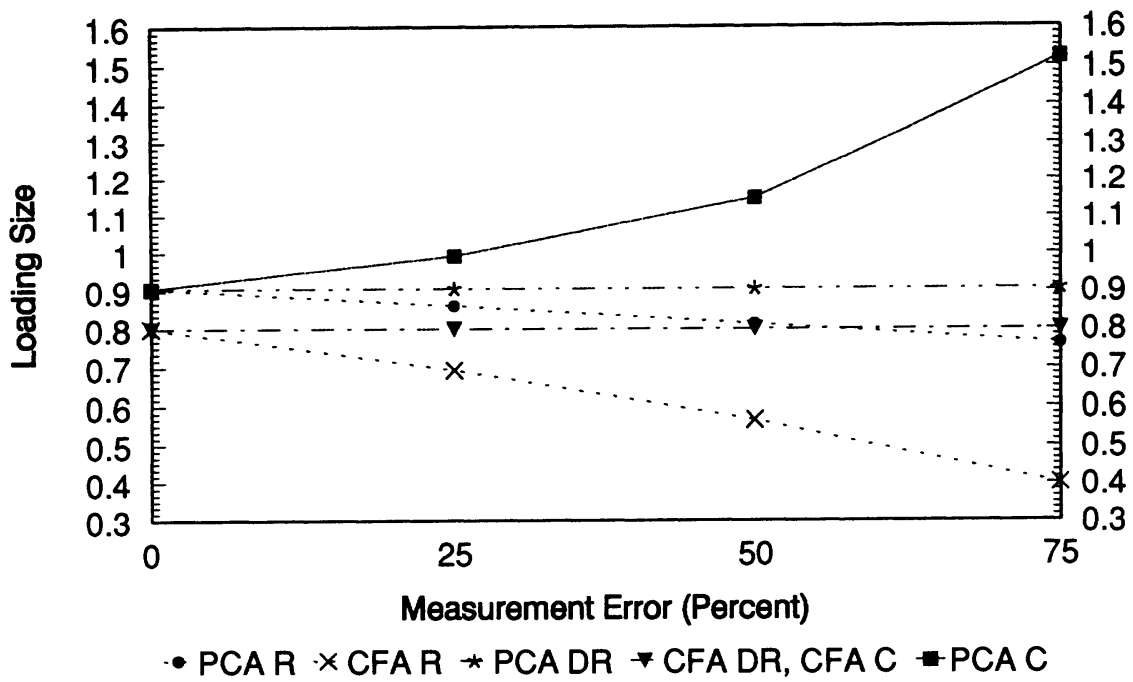

FIGURE 1. Calculated factor loading size as a function of percentage of measurement error and type of matrix factors are to be extracted from $(R=$ correlation matrix, $D R=$ disattenuated correlation matrix, $C=$ covariance matrix). Number of variables $=10$, number of factors $=5$ (i.e., $\mathrm{m}=2$ ), and unattenuated factor loadings $=.8$.

\section{Method}

The estimated factor loadings for CFA and PCA were computed for various combinations of unattenuated factor loading size, number of variables, number of factors, and percentage of measurement error. These values were entered into Equations (3), (4), (5), (6), and (7), and the resulting factor loadings could be compared to the known unattenuated correlations between variables and latent factors. For all calculations, the number of factors was kept constant at five.

For example, in some of the conditions presented, we postulated that the unattenuated factor loading size was .8 , and the percentage of measurement error 25 . In this case, each estimated communality was $.8^{2}(1-.25)$, or .48 . This value was then substituted for $\hat{h}^{2}$ in Equation (3), and summed over $p$, resulting the value $\hat{\sigma}_{\text {CFAcom }}^{2}$. Following this, combinations of Equations (4), (5), (6), and (7) were employed to compute the CFA and PCA loadings for any combination of $r$ and $p(m=p / r$; i.e., $m$ represents the number of indicators per factor). These steps were carried out for other values of unattenuated factor loading size, percentage of measurement error, $r$, and $p$. Decomposition of the correlation matrix, the disattenuated correlation matrix, and the covariance matrix were considered.

For the conditions involving the covariance matrix, the total variance is arrived at in two steps. First, the variance attributable to specific variance $\left(p\left[1-\lambda_{u}^{2}\right)\right]$, where $\lambda_{u}^{2}$ is the squared unattenuated factor loading) is added to the 


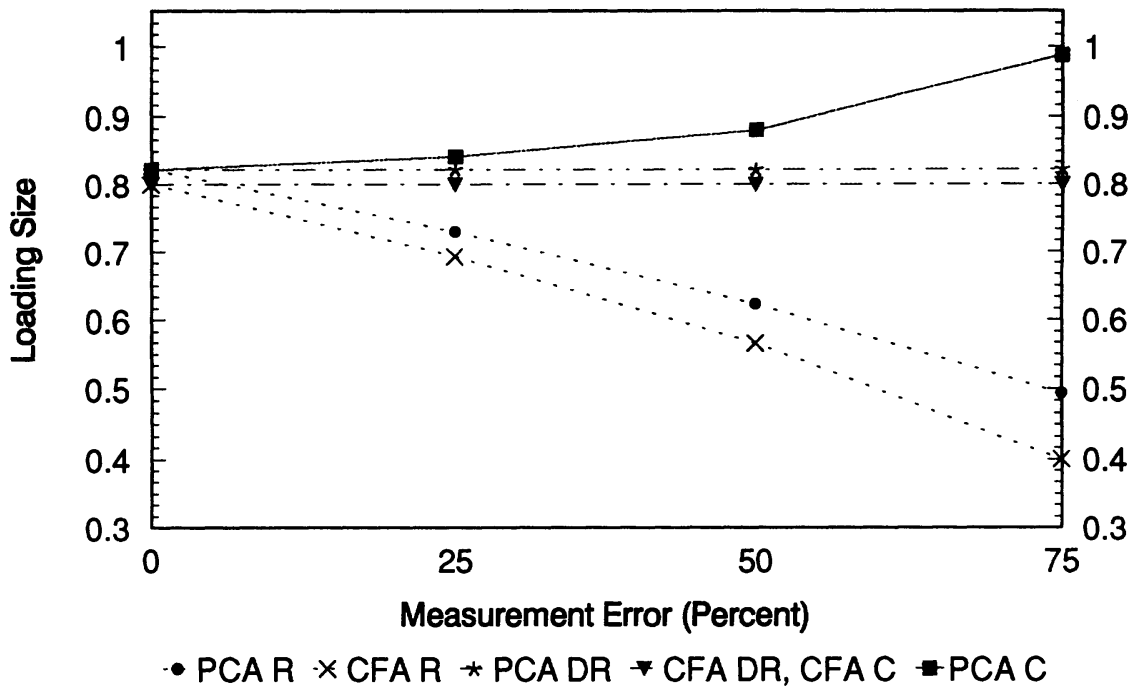

FIGURE 2. Calculated factor loading size as a function of percentage of measurement error and type of matrix factors are to be extracted from $(R=$ correlation matrix, $D R=$ disattenuated correlation matrix, $C=$ covariance matrix). Number of variables $=50$, number of factors $=5$ (i.e., $\mathrm{m}=10$ ), and unattenuated factor loadings $=.8$.

CFA estimated common variance. Second, variance attributable to measurement error is added. For example, in the $50 \%$ measurement error conditions, the true score variance, or the variance due to specific and common variance, was doubled to produce the total observed variance.

\section{Results \\ Unadjusted Correlation Matrix}

CFA

Figures 1 to 4 show the consistently attenuated loadings provided by CFA when the factors are extracted from the unadjusted correlation matrix (relative to the unattenuated factor loadings), except in the unrealistic situation where there is no measurement error in the data. Although CFA loadings are accurate estimates of the attenuated correlations between the observed variables and the factors, they cannot be considered accurate estimates of the unattenuated correlations between the observed variables and the factors. This is due to the fact that, when measurement error is present, the CFA estimated common variance will be lower than the unattenuated common variance.

Figure 5 shows the relationship between percentage of measurement error and unattenuated factor loading size, when measuring the difference between the unattenuated and estimated factor loading size. We see that as percentage of measurement error and unattenuated loading size increases, differences increase. 


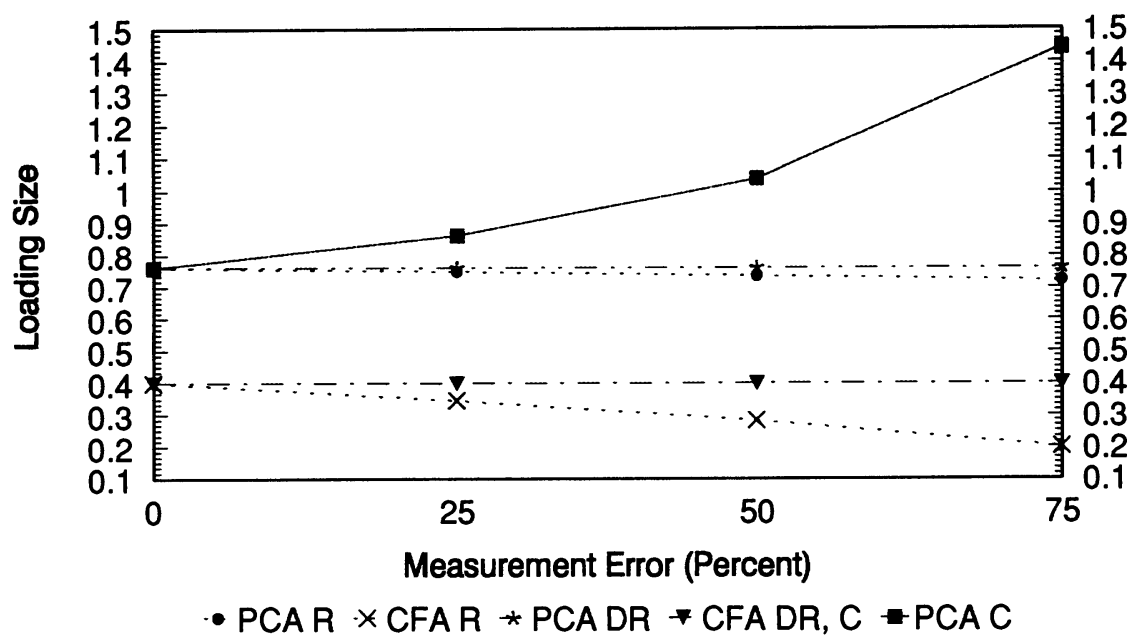

FIGURE 3. Calculated factor loading size as a function of percentage of measurement error and type of matrix factors are to be extracted $(R=$ correlation matrix, $D R=$ disattenuated correlation matrix, $C=$ covariance matrix). Number of variables $=10$, number of factors $=5$ (i.e., $\mathrm{m}=2$ ), and unattenuated factor loadings $=.4$.

Because these loading estimate differences are based on common variance estimate differences, the percentage of attenuation, for any unattenuated factor loading size or value of $m$, may be computed as:

$$
1-\frac{\sqrt{\hat{h}^{2}}}{\sqrt{h^{2}}},
$$

where $h^{2}$ is the unattenuated communality, and $\hat{h}^{2}$ the estimated communality. Thus, at $25 \%$ measurement error, and unattenuated factor loading size of .8 , the percentage of attenuation will equal $13.4\left(1-\frac{\sqrt{.48}}{\sqrt{.64}}\right.$, where $\left.\hat{h}^{2}=.8^{2}[1-.25]\right)$. $P C A$

PCA loadings will be overestimates of the attenuated correlations between the observed variables and the factors. As pointed out by Widaman $(1990,1993)$, this situation occurs because the PCA estimated common variance is larger than that of CFA. Thus, under these conditions, PCA may seem a better candidate for estimation of the unattenuated common variance and factor loadings than CFA, which would produce attenuated estimates. However, this effect results from a bias in estimating attenuated loading, which behaves rather unpredictably. In fact, PCA loadings may be higher or lower than the unattenuated factor loadings, depending on data characteristics (see Figures 1 to 4 ).

This fluctuating performance of PCA is based in a three-way interaction between unattenuated factor loading size, percentage of measurement error, and 


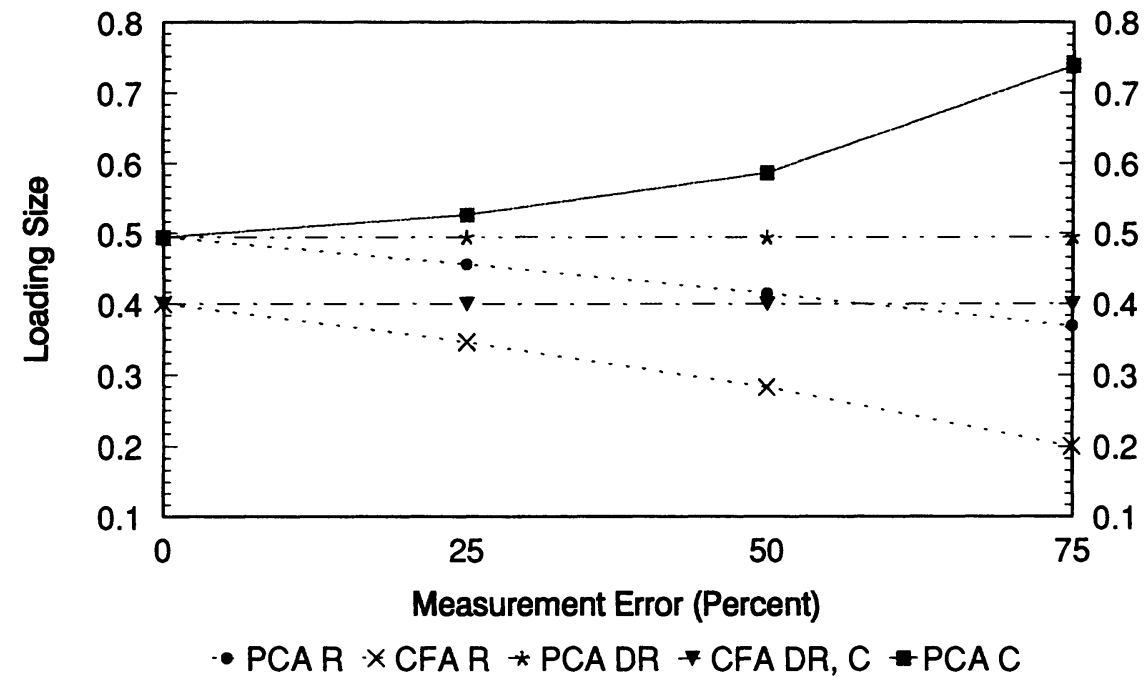

FIGURE 4. Calculated factor loading size as a function of percentage of measurement error and type of matrix factors are to be extracted from $(R=$ correlation matrix, $D R=$ disattenuated correlation matrix, $C=$ covariance matrix). Number of variables $=50$, number of factors $=5$ (i.e., $\mathrm{m}=10$ ), and unattenuated factor loadings $=.4$.

the value for $m$. Calculation of $\hat{\sigma}_{P C A c o m}^{2}$, through re-arrangement of Equations (5) and (6), demonstrates this interaction:

$$
\hat{\sigma}_{\text {PCAcom }}^{2}=\hat{\sigma}_{\text {CFAcom }}^{2}+\frac{r}{p}\left(\sigma_{\text {total }}^{2}-\hat{\sigma}_{\text {CFAcom }}^{2}\right),
$$

where the term $\sigma_{\text {total }}^{2}-\hat{\sigma}_{\text {CFAcom }}^{2}$ reflects both specific and error variance. Thus, due to this complex interaction of effects, in most situations it would be very difficult to predict how accurately PCA loadings would estimate the unattenuated factor loadings.

\section{Disattenuated Correlation Matrix}

CFA

Figures 1 to 4 show that CFA accurately estimates the unattenuated factor loadings when the factors are extracted from the disattenuated correlation matrix. This is due to the fact that, under these conditions, the matrix resulting from $R-U^{2}$ (see Equation [1]) would be composed only of unattenuated common variance. That is to say, the CFA estimated common variance $\left(\hat{\sigma}_{C F A c o m}^{2}\right)$ would be equal to the unattenuated common variance, resulting in accurate estimates of the unattenuated factor loadings (see Equations [3] and [4]. 


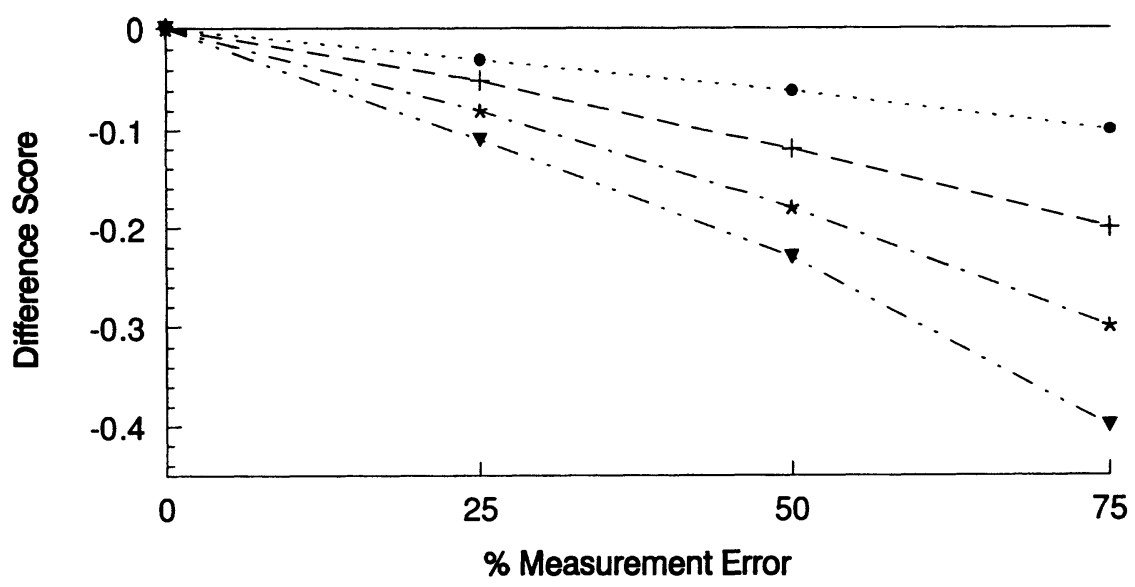

- Unattenuated Loading $=.8$ + Unattenuated Loading $=.6$

* Unattenuated Loading $=.4 *$ Unattenuated Loading $=.2$

FIGURE 5. Attenuation of CFA loadings, when correlation matrix is decomposed, as a function of percentage of measurement error and unattenuated factor loading size. The ordinate values represent the difference between the CFA estimated loading and the unattenuated factor loading.

$P C A$

When the factors are extracted from the disattenuated correlation matrix using PCA, the unattenuated factor loadings are consistently overestimated (see Figures 1 to 4). Unlike the situation where the correlation matrix was decomposed, these estimates are not influenced by measurement error. They are influenced by unattenuated factor loading size, and the value of $m$. Referring to Equation (9), the term $\sigma_{\text {total }}^{2}-\hat{\sigma}_{C F A c o m}^{2}$ now reflects only specific variance, rather than specific and error variance (the latter was the situation when the correlation matrix was decomposed). If there is little specific variance in the data (i.e., the unattenuated factor loading size is large), and a large value of $m$ (i.e., $\frac{r}{p}$ is small), we observe little difference between CFA and PCA coefficients (see Figure 6). Diminishment of inter-procedural differences with increases in number of indicators per factor $(m)$ is a well known effect, and has been previously reported (Borgatta et al., 1986; McArdle, 1990; Snook \& Gorsuch, 1989; Widaman, 1990, 1993).

\section{Covariance Matrix}

CFA

Figures 1 to 4 show that CFA provides accurate estimates of the unattenuated factor loadings when factors are extracted from the covariance matrix. As was the case for CFA on the disattenuated correlation matrix, under these conditions, the matrix resulting from $R-U^{2}$ (see Equation [1]) would be composed only of 


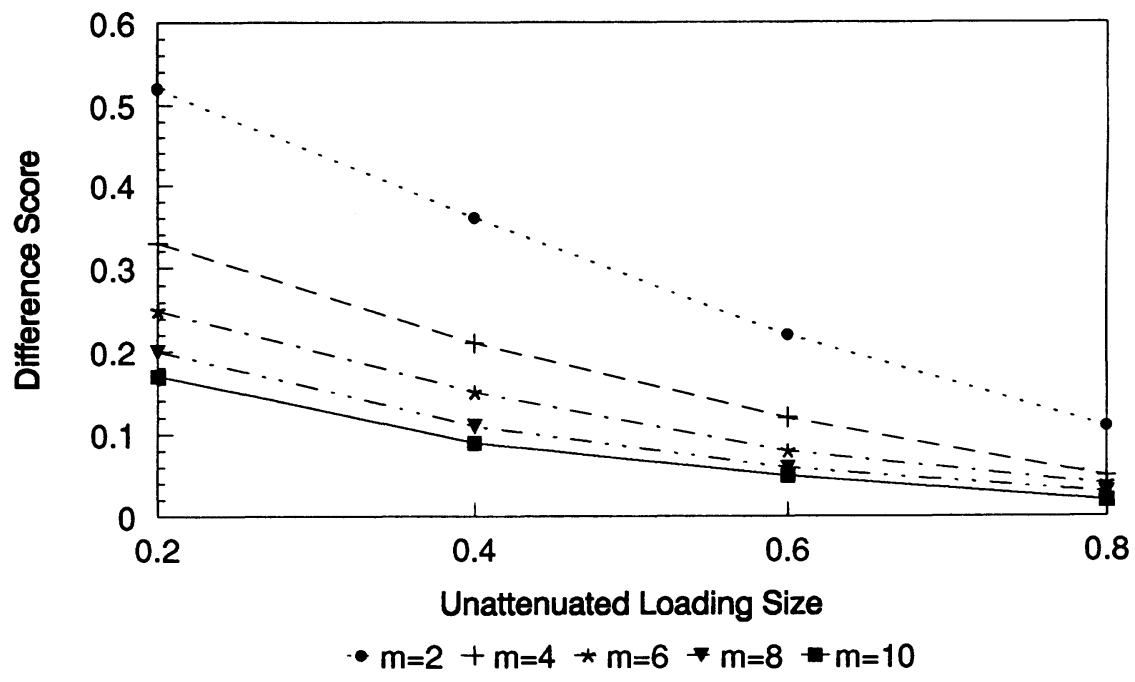

FIGURE 6. PCA overestimates of unattenuated factor loading size, when the disattenuated correlation matrix is decomposed, as a function of number of indicators per factor (m) and unattenuated factor loading size. The ordinate values represent the difference of the PCA estimated factor loading and the unattenuated factor loading.

unattenuated common variance. That is to say, the CFA estimated common variance $\left(\hat{\sigma}_{\text {CFAcom }}^{2}\right.$ ) would be equal to the unattenuated common variance, resulting in accurate estimates of the unattenuated factor loadings. However, CFA produces loadings that are covariances between variables and factors, as opposed to the traditionally interpreted correlations.

$P C A$

When factors are extracted from the covariance matrix, PCA would overestimate the unattenuated factor loadings in all conditions. As was the case when the unadjusted correlation matrix was used as input, these coefficients are influenced by a three-way interaction between percentage of measurement error, specific variance, and the value of $m$. Again referring to Equation (9), the term $\sigma_{\text {total }}^{2}-\hat{\sigma}_{\text {CFAcom }}^{2}$ reflects specific and error variance when the covariance matrix is decomposed, as both contribute to the size of the total variance (i.e., the sum of the diagonal elements of the covariance matrix).

\section{Discussion}

Traditional exploratory factor analytic techniques (viz., PCA and CFA used to extract factors from the attenuated correlation matrix) cannot reliably estimate unattenuated factor loadings if measurement error is present in the data. CFA consistently produces underestimates in these conditions, and PCA produces both under- and overestimates, in a complex, difficult-to-predict pattern. Alter- 
natively, when the disattenuated correlation matrix or the covariance matrix is decomposed, CFA can produce accurate estimates of the unattenuated factor loadings. PCA, on the other hand, produces overestimates in both these conditions. Moreover, the overestimates produced by PCA in conjunction with the covariance matrix are greater than those produced in conjunction with the disattenuated correlation matrix, due to differential influences of measurement error. The basis for these differences in exploratory factor analytic methodology is based in differences in common variance estimates, as in demonstrated in Equations (3) to (7).

We have simulated some extreme situations that would not, or at least should not, occur in a real-world research setting. For example, most researchers would not consider using measures containing $75 \%$ measurement error, or interpreting a factor analytic solution where the factors are defined by only two indicators. We simulated these conditions for pedagogical purposes, but we cannot recommend drawing conclusions from research being carried out under these conditions, despite the fact that, in theory, our equations would apply to the resulting estimates.

Note that if the percentage of measurement error is small, and the number of indicators per factor large, the advantage of using the disattenuated correlation matrix, and the differences between CFA and PCA, are greatly reduced. However, the importance of recognizing potential interactions between factor analytic methodology and correlation attenuation due to measurement error should not be underestimated. For example, in traditional research settings, reliabilities as low as .70 are considered adequate (Nunnally \& Bernstein, 1994, p. 265). Application of Equations (3) to (7) demonstrates that, for a data set with $30 \%$ measurement error, and ten indicators per factor, traditional (i.e., correlation matrix used as input) CFA loadings would be attenuated by $16 \%$, while PCA loadings would be $13 \%$ higher than the unattenuated values. With four indicators per factor, PCA estimates would be $44 \%$ higher than the unattenuated values, while CFA estimates would remain attenuated by $16 \%$.

The above example focused on use of the unadjusted correlation matrix as input. This may be the most commonly encountered situation, because practical complications often render disattenuation of the correlation matrix impossible. Reliability values may not be available, or may not be accurate, as large sample sizes are needed to produce accurate reliability estimates (Hakstian, Schroeder, \& Rogers, 1988, recommend a sample size of at least 150). Inaccurate reliability estimates may lead to over- or undercorrection errors (and possibly adjusted correlations greater than 1). Although reliability estimates may not be available, the equations presented in this paper can still provide a framework on which researchers can assess how measurement error may be interacting with their choice of factor analytic methodology. Moreover, if reliability estimates are unavailable, researchers can extract factors from the covariance matrix, and accurately estimate unattenuated factor loadings using CFA. Interpretation may seem awkward if the scale of the variables is not uniform; however, covariances 


\section{Woodward and Hunter}

can be standardized to the scale of any of the measures, and the loadings interpreted in a relative sense.

In accordance with measurement theory, estimation of unattenuated factor loadings is highly desirable. The neglect of these parameters in previous discussions of factor analytic methodology was unnecessary, and may have led to errors of inference. For example, as the variance of the percentage of measurement error over variables increases, the further the shape of the standardized observed-score space diverges from that of the true-score space. Accordingly, the loading matrix derived from analysis of the observed scores may differ greatly from what would be derived from analysis of the true scores. In this manuscript we have shown that unattenuated factor loadings are retrievable. Researchers are encouraged to use the disattenuated correlation matrix, or the covariance matrix, as input to CFA algorithms for accurate estimation of these parameters. If accurate reliability estimates are not available, and use of a covariance matrix is undesirable, the information presented here provides a framework for dimension reduction method choice, demonstrating the effect of specific data characteristics, such as measurement quality, number of indicators per factor, and unattenuated loading size.

\section{References}

Bentler, P. M., \& Kano, Y. (1990). On the equivalence of factors and components. Multivariate Behavioral Research, 25, 67-74.

Bookstein, F. L. (1990). Least squares and latent variables. Multivariate Behavioral Research, 25, 75-80.

$\rightarrow$ Borgatta, E. F., Kercher, K., \& Stull, D. E. (1986). A cautionary note on the use of principal components analysis. Sociological Methods and Research, 15, 160-168.

Browne, M. W. (1968). A comparison of factor analytic techniques. Psychometrika, 33, 267-334.

Fava, J. L., \& Velicer, W. F. (1992). An empirical comparison of factor, image, component, and scale scores. Multivariate Behavioral Research, 27, 301-322.

Gorsuch, R. L. (1983). Factor Analysis. Hillsdale, NJ: Lawrence Erlbaum.

$\rightarrow$ Gorsuch, R. L. (1990). Common factor analysis versus component analysis: some well and little known facts. Multivariate Behavioral Research, 25, 33-39.

Hakstian, A. R., Schroeder, M. L., \& Rogers, T. (1988). Inferential procedures for correlation coefficients corrected for attenuation. Psychometrika, 53(1), 27-43.

$\rightarrow$ Hotelling, H. (1933). Analysis of a complex of statistical variables into principal components. Journal of Educational Psychology, 24, 417-441, 498-520.

$\rightarrow$ Kaiser, H. F. (1970). A second generation little jiffy. Psychometrika, 35, 401-415.

McArdle, J. J. (1990). Principles versus principals of structural factor analyses. Multivariate Behavioral Research, 25, 81-87.

Mulaik, S. A. (1990). Blurring the distinction between component analysis and common factor analysis. Multivariate Behavioral Research, 25, 53-59.

Nunnally, J. C., \& Bernstein, I. H. (1994). Psychometric Theory: Third Edition. New York: McGraw-Hill.

Ree, M. J., \& Earles, J. A. (1992). Intelligence is the best predictor of job performance. Current Directions in Psychological Science, 1, 86-89. 
Rozeboom, W. W. (1990). Whatever happened to broad perspective? Multivariate Behavioral Research, 25, 61-65.

Snook, S. C., \& Gorsuch, R. L. (1989). Component analysis versus common factor analysis: a Monte Carlo study. Psychological Bulletin, 106, 148-154.

Spearman, C. (1904). The proof and measurement of association between two things. American Journal of Psychology, 15, 72-101.

Thurstone, L. L. (1935). The Vectors of Mind. Chicago: University of Chicago Press.

Velicer, W. F., \& Fava, J. L. (1987). An evaluation of the effects of variable sampling on component, image, and factor analysis. Multivariate Behavioral Research, 22, 193-209.

Velicer, W. F., \& Jackson, D. N. (1990a). Component analysis versus common factor analysis: some issues in selecting an appropriate procedure. Multivariate Behavioral Research, 25, 1-28.

Velicer, W. F., \& Jackson, D. N. (1990b). Component analysis versus common factor analysis: some further observations. Multivariate Behavioral Research, 25, 97-114.

Velicer, W. F., Peacock, A. C., \& Jackson, D. C. (1982). A comparison of component and factor patterns: a Monte Carlo approach. Multivariate Behavioral Research, 17, 371-388.

Widaman, K. F. (1990). Bias in pattern loadings represented by common factor analysis and component analysis. Multivariate Behavioral Research, 25, 89-95.

Widaman, K. F. (1993). Common factor analysis versus principal component analysis: Differential bias in representing model parameters? Multivariate Behavioral Research, $28,263-311$.

\section{Authors}

TODD S. WOODWARD is a post-doctoral fellow in the Department of Psychology, University of British Columbia 2136, West Mall, Vancouver, British Columbia, Canada V6T 1Z4; twoodward@cortex.psych.ubc.ca. His research interests include cognitive neuropsychology and multivariate statistical methodology.

MICHAEL A. HUNTER is an Associate Professor at the Department of Psychology, University of Victoria, P. O. Box 3050, Victoria, British Columbia, Canada V8W 3P5; mhunter@uvic.ca. His teaching and research interests include randomization/permutation theory and analysis, and psychometrics. 